\title{
Compact Superconducting Magnet for Linear Accelerators
}

\author{
V. S. Kashikhin, N. Andreev, N. Kimura, Y. Orlov, M. A. Tartaglia, A. Yamamoto
}

\begin{abstract}
New Linear Accelerators based on SCRF cavities need compact and efficient superconducting magnet packages to focus and steer electron or proton beams. These magnets should be combined with SCRF cryomodules and installed inside or between them. A recent activity in this area was directed by FNAL-KEK collaboration to splittable conduction cooled magnets. Several magnet prototypes were built and successfully tested. These magnets were designed for high energy beams used in ILC, and Project-X. Nevertheless, there is an interest to explore splittable conduction cooled magnets for new accelerators: FNAL ASTA and PIP-II, KEK STF, SLAC LCLS II. The paper describes a conceptual design of splittable conduction cooled superconducting magnet which could be mounted inside SCRF cryomodule. The magnet package combines the quadrupole magnet and dipole correctors. The presented magnetic design confirms the specified magnet package parameters.
\end{abstract}

Index Terms-Accelerator, cryomodule, linac, magnet, superconducting.

\section{INTRODUCTION}

$\mathrm{N}$ EW Linear Accelerators are based on the superconducting RF and magnet technology. Besides large linear colliders like ILC [1]-[2], there are now a number of smaller linear accelerators under design and construction: FNAL ASTA and PIP-II, KEK STF, SLAC LCLS-II.

In recent years there were proposed and investigated several magnets for linear accelerators having the splittable configuration and conduction cooling [3]-[5]. These magnets could be installed in the cryomodule (See Fig. 1) after the final assembly of SCRF cavities string in the clean room. The magnet package does not have the LHe vessel and is conductively cooled by thermal sinks connected to the LHe supply pipe. The first splittable ILC magnet prototype was built and successfully tested by FNAL-KEK collaboration (See Fig. 2). The magnet showed good performance and met very tight specifications related to the quadrupole magnetic center stability [6] - [7] which is very critical for the long ILC Linac with large number of cryomodules. For the lower energy Linacs than ILC the needed magnet package strength is

Manuscript received 10 August 2014. This work was supported in part by Fermi Research Alliance, LLC, under contract No. DE-AC02-07CH11359 with the U.S. Department of Energy, and in part by the Japan-U.S. cooperative program in High Energy Physics.

V. S. Kashikhin\#, N. Andreev, M. Tartaglia are with the Fermi National Accelerator Laboratory, Batavia, IL 60510, USA (corresponding author\# phone: 630-840-2899; fax: 630-840-6766; e-mail: kash@fnal.gov).

N. Kimura, A. Yamamoto are with KEK, Japan. order of magnitude lower. It opens the way to build even more compact splittable conduction cooled magnets.

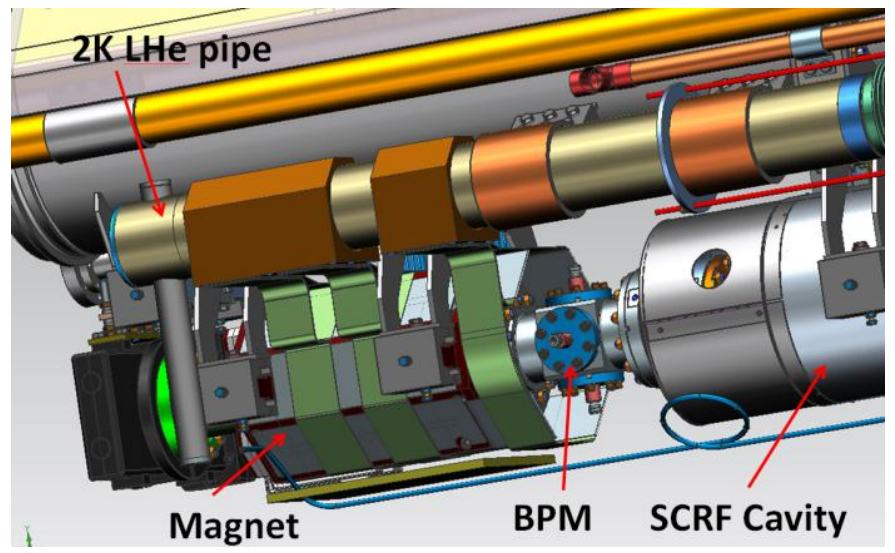

Fig. 1. Magnet package inside the SCRF Cryomodule.

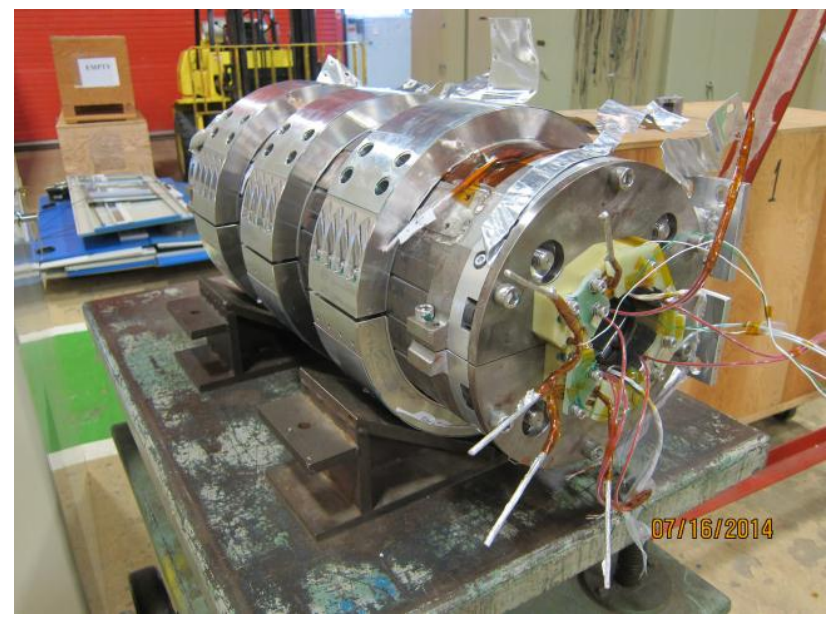

Fig. 2. Splittable conduction cooled magnet for ILC.

\section{QUADRUPOLE MAGNET DESIGN}

Magnet package specifications for new linear accelerators are shown in Table 1 . One could see that the peak ILC quadrupole magnet integrated gradient $36 \mathrm{~T}$ is 7-18 times larger than for other machines under the design now. But because the magnet has aperture defined by the aperture of SCRF cavity, the magnet length could not be reduced proportionally in order to keep the field quality reasonable. The magnet good field volume is small relative to the pole tip distance, and this substantially reduces demands on the magnet fabrication, and assembly tolerances. 
TABLE I

MAGNET PACKAge SPECIFICATIONS

\begin{tabular}{lclcc}
\hline \hline \multicolumn{1}{c}{ Parameter } & ILC & XFEL & $\begin{array}{c}\text { ASTA/ } \\
\text { KEK }\end{array}$ & LCLS-II \\
& & & 3.0 & 2.0 \\
\hline $\begin{array}{l}\text { Integrated peak gradient, T } \\
\text { Aperture, mm }\end{array}$ & 36 & 5.2 & 78 & 78 \\
Effective length, mm & 660 & 195 & 190 & 150 \\
$\begin{array}{l}\text { Peak gradient, T/m } \\
\text { Peak current, A }\end{array}$ & 54 & 26.7 & 26.7 & 13.3 \\
$\begin{array}{l}\text { NbTi superconductor dia., } \\
\text { mm }\end{array}$ & 0.5 & 0.55 & 50 & 50 \\
$\begin{array}{l}\text { Superconductor filament } \\
\text { size, } \mu \text { m }\end{array}$ & 3.7 & 6.0 & 3.7 & 0.5 \\
$\begin{array}{l}\text { Dipole corrector integrated } \\
\text { strength, T-m }\end{array}$ & 0.075 & 0.009 & 0.005 & 0.005 \\
$\begin{array}{l}\text { Max magnetic center offset } \\
\text { in cryomodule, mm }\end{array}$ & 0.3 & 0.5 & 0.5 & 0.5 \\
$\begin{array}{l}\text { Total length, mm } \\
\text { Quantity required }\end{array}$ & 800 & 300 & 325 & 250 \\
\hline \hline
\end{tabular}

The compact quadrupole magnetic design was based on the ILC, and ASTA/KEK quadrupole magnet designs (See Fig. 2Fig. 4). There were correspondingly reduced magnet coil and yoke dimensions to obtain $2 \mathrm{~T}$ for the compact magnet integrated strength. The magnet field was calculated by OPERA3d. Fig. 3 shows the quadrupole geometry and the iron core flux density.

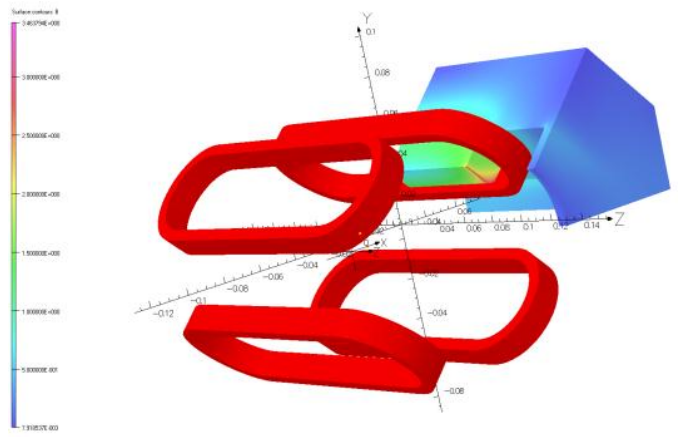

Fig. 3. The quadrupole magnet simulated geometry.

Because SCRF cavity is placed near the magnet, and is very sensitive to the external magnetic field, the quadrupole has ferromagnetic end field clamps to reduce the fringe field as much as possible. At the magnet pole tip distance of $90 \mathrm{~mm}$, and $120 \mathrm{~mm}$ pole length the magnetic field has large amount of end fields as shown in Fig. 4.

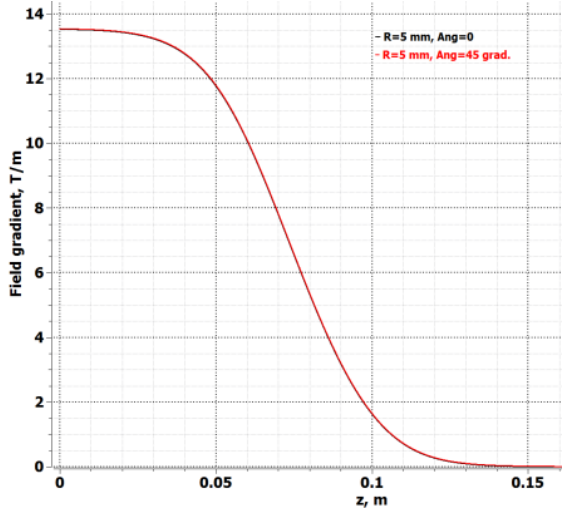

Fig. 4. Quadrupole field gradient along the beam path.

End fields non-linearity is difficult to compensate by the magnet iron yoke configuration. Nevertheless, at the $10 \mathrm{~mm}$ diameter of the good field area, the integrated quadrupole gradient homogeneity has a reasonable value of $0.17 \%$.

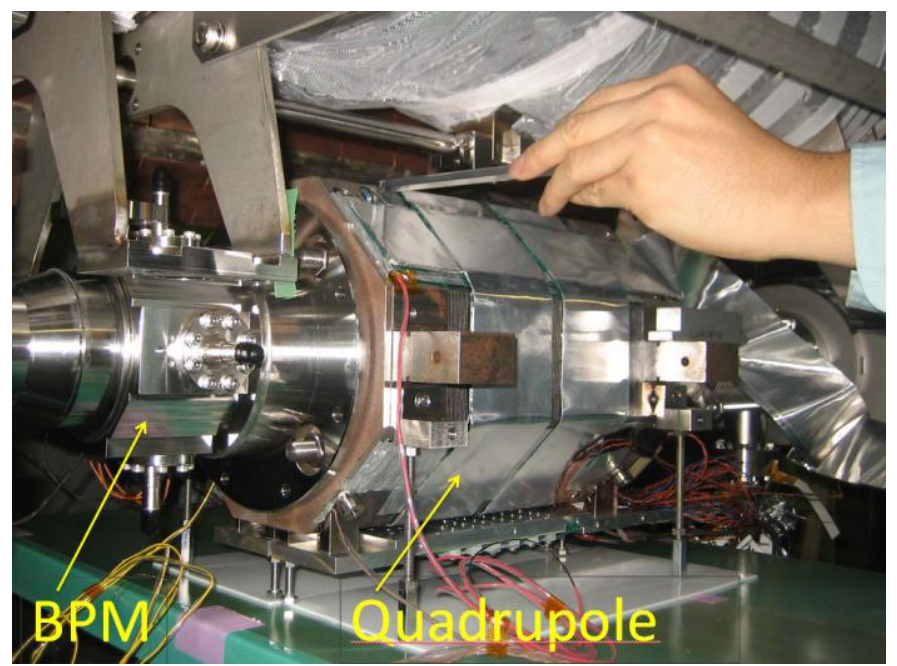

Fig. 5. Splittable conduction cooled quadrupole in the KEK Cryomodule. BPM- beam position monitor is rigidly attached to quadrupole plate.

\section{SEPARATE DipOlE CORRECTORS}

The magnet package should include vertical and horizontal dipole correctors. There are two options: combine correctors with quadrupole coils or separate them from the quadrupole. The first option was investigated for ILC, and XFEL. It was found rather large cross-talk between magnets due to the superconductor magnetization effects for ILC [9], and XFEL [10]. In the ILC magnet with combined dipole correctors there is rather strong coupling through the superconductor magnetization which increases the quadrupole magnetic center shift during the quadrupole strength change [9]. The XFEL quadrupole magnet has dipole shell type coils placed close to the aperture. This increases the influence of superconductor magnetization effects. These effects are strong at low field and difficult to manage. Superconductor heating above Tc before the working cycle, and a proper degaussing may help to some extent; This was done in the LHC combined function correctors. Another approach is to separate correctors from the quadrupole [11] but also make them splittable with conduction cooling (See Fig. 6).

The short quadrupole length allows extra space to place the separate dipole corrector. Fig. 6 shows the simulated combined vertical (VD), and horizontal (HD) dipole correctors geometry. The conventional shell type coils generating the horizontal dipole field do not cross the vertical magnet split plane and could be used for the dipole corrector. But for the vertical dipole field coils should be configured in such a way to not cross the split plane as shown in Fig.5. In this case each magnet quadrant has three coil sections connected in series. Number of section turns was optimized using OPERA2D Optimizer code to obtain the good field homogeneity in the magnet aperture. 


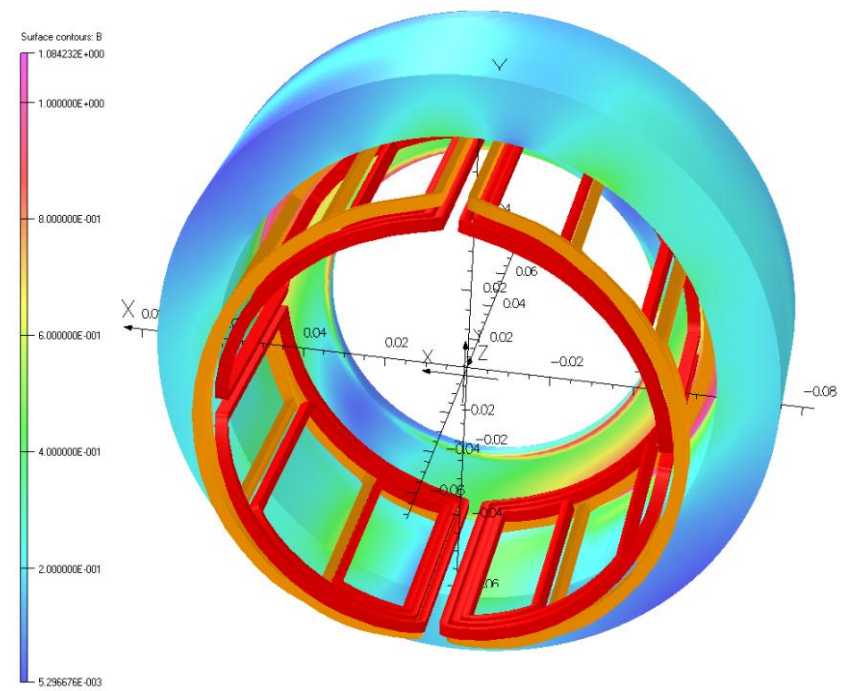

Fig. 6. Separate combined vertical and horizontal dipole corrector.

Nevertheless, it should be noted that the horizontal dipole has more natural shell type configuration and generates a more homogeneous integrated field than the vertical dipole (See Fig. 4 - Fig. 5). The magnet could be rotated $90^{\circ}$ to provide better field homogeneity for the vertical field corresponding to beam steering in the horizontal plane.

All VD and HD coils are wound using $0.5 \mathrm{~mm} \mathrm{NbTi}$ superconductor in the aluminum mandrel slots. Coils and mandrel geometry are shown in Fig. 6 - Fig. 7.

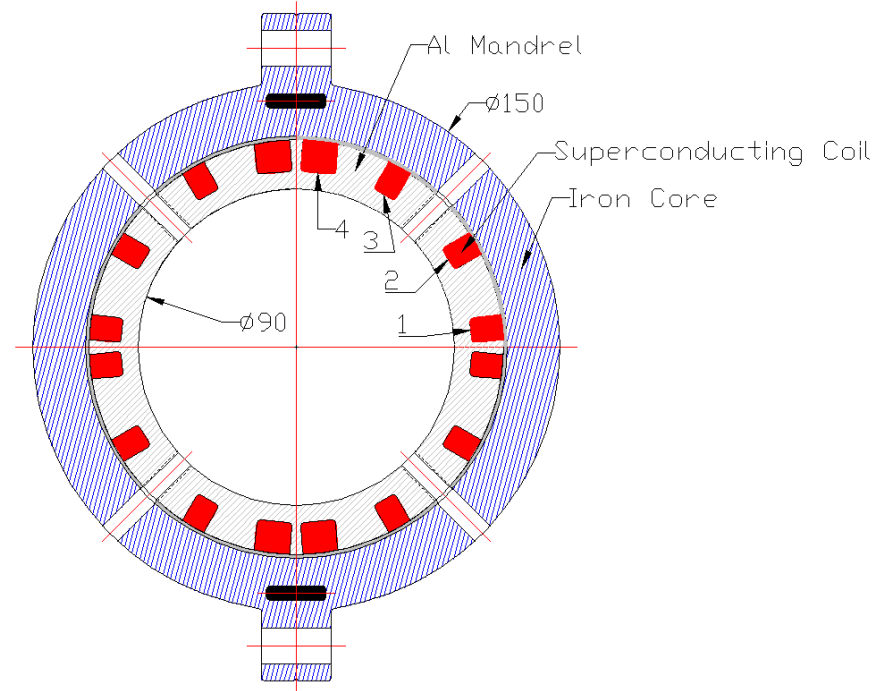

Fig. 7. Dipole corrector cross-section

Number of turns and the current directions for each slot (See Fig. 7) are shown in Table 2. Mandrel slots covered by a Kapton film with an adhesive coating works as electrical ground insulation. Coils in the mandrel are vacuum impregnated with epoxy. Each half of the coil block is bolted to the yoke half core. Both quadrupole and dipole halves are initially mounted around the beam pipe, and rigidly bolted to each other to form a magnetically and mechanically stable unit.

The main drawback of separate quadrupole and corrector designs is that the magnet package total length will increase by about $100 \mathrm{~mm}$.
TABLE II

CORRECTOR COIL TURNS IN SLOTS

\begin{tabular}{ccc}
\hline \hline Slot N & VD turns & HD turns \\
\hline 1 & 38 & 0 \\
2 & 22 & 24 \\
3 & 75 & 40 \\
4 & $-135^{*}$ & 26
\end{tabular}

* Minus sign shows return conductor turns with opposite direction of current.

The number of turns in each slot is defined by the vertical and horizontal dipole field symmetry. Table 3 shows the magnet package parameters for the $2 \mathrm{~T}$ peak quadrupole strength with the separated dipole correctors.

TABLE III

SEPARATED MAGNETS PARAMETERS

\begin{tabular}{lcc}
\hline \hline \multicolumn{1}{c}{ Parameter } & Quadrupole & $\begin{array}{c}\text { Dipole } \\
\text { VD/HD }\end{array}$ \\
\hline Peak operating current, A & 50 & 50 \\
Magnet total length, mm & 220 & 100 \\
SC wire diameter, mm & 0.5 & 0.5 \\
NbTi filament size, $\mu \mathrm{m}$ & 3.7 & 3.7 \\
Cu:Sc ratio & 1.5 & 1.5 \\
SC critical current at 5 T, A & 200 & 200 \\
Peak coil field at 50 A current, T & 0.94 & 0.67 \\
Magnetic field stored energy, J & 135 & $6.2 / 5.2$ \\
Inductance, mH & 107 & $5.0 / 4.1$ \\
Coil number of turns/pole & 220 & $135 / 90$ \\
Yoke outer diameter, mm & 200 & 150 \\
\hline \hline
\end{tabular}

\section{COMBIned QuAdRupole WITH DipOle CORRECTORS}

It was shown in the previous section that it is possible to make $100 \mathrm{~mm}$ long separate splittable dipole corrector. But it is still is very attractive option to combine quadrupole and dipole into common racetrack coils (See Fig. 8).

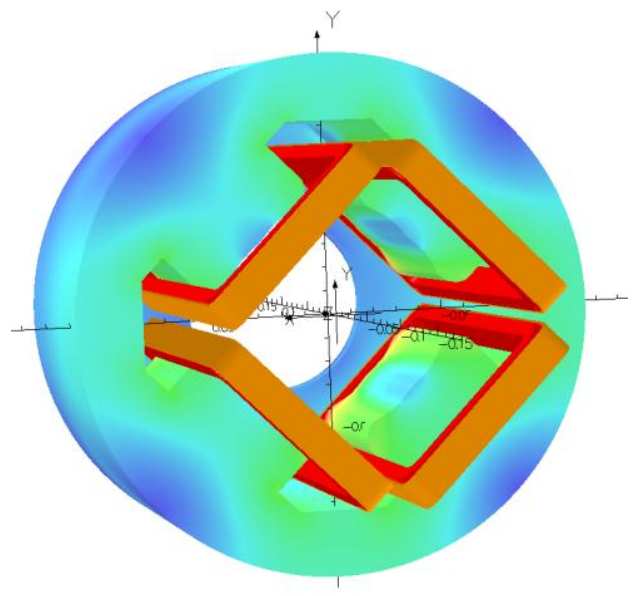


Fig. 8. Combined quadrupole and dipole correctors.

The combined magnet package dipole field distribution is shown in Fig.9. In spite of the short pole length and quadrupole reasonable field quality was obtained in the $10 \mathrm{~mm}$ diameter good field area: $0.14 \%$ for the quadrupole, and $1.1 \%$ for dipoles. Better results could be achieved by the pole profile and pole ends optimization.

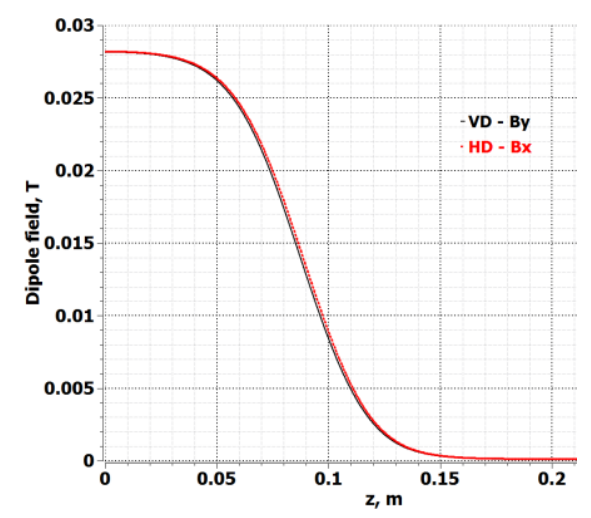

Fig. 9. Dipole correctors field along the beam path at $50 \mathrm{~A}$ currents.

The magnet parameters for $2 \mathrm{~T}$ quadrupole peak strength are shown in Table 4. It has only $250 \mathrm{~mm}$ length, small number of coil turns, and correspondingly low stored energy. So, the magnet coils could be protected during a quench by a $10 \Omega$ external dump resistor.

TABLE IV

COMBINED MAGNETS PARAMETERS

\begin{tabular}{|c|c|c|}
\hline Parameter & Quadrupole & $\begin{array}{l}\text { Dipole } \\
\text { VD/HD }\end{array}$ \\
\hline Peak operating current, $\mathrm{A}$ & 50 & 50 \\
\hline Magnet total length, mm & \multicolumn{2}{|c|}{250} \\
\hline Peak coil field at 50 A current, $\mathrm{T}$ & 1.1 & 0.1 \\
\hline Magnetic field stored energy, $\mathrm{J}$ & 206 & 1.2 \\
\hline Inductance, $\mathrm{mH}$ & 212 & 1.05 \\
\hline Coil number of turns/pole & 176 & $18 / 18$ \\
\hline Yoke outer diameter, mm & \multicolumn{2}{|c|}{220} \\
\hline
\end{tabular}

Because the number of corrector turns is about 10 times less than for the quadrupole, and these turns are far away from the magnet aperture their negative effect on the field will be low. Besides all coils are mounted inside windows of the iron dominated magnet, and the iron in the main part reduces the coil influence on the field. Dipole coils are wound on top of the quadrupole coils and connected in a way to form the vertical and horizontal dipole fields. The influence of superconductor magnetization was investigated with OPERA2d code with the approach used in [12]. Simulations for the whole range of operating currents found a negligible effect for the main quadrupole field distortions. Fig. 10 shows flux lines from the superconductor persistent currents, and field distortions in the aperture which are less than $10^{-6}$.

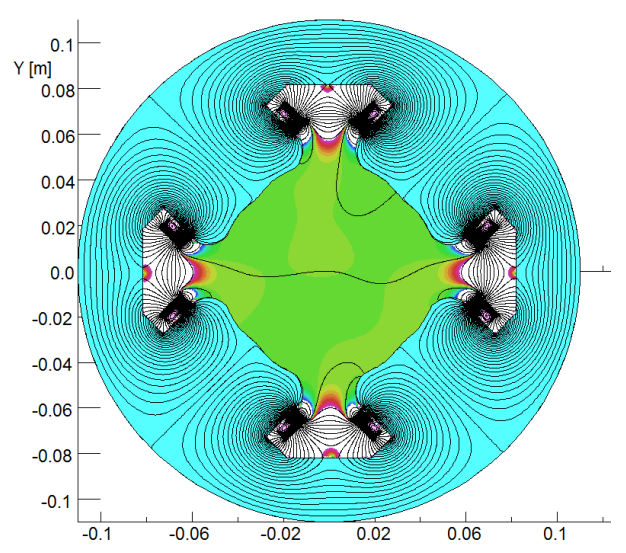

Fig. 10. Superconductor magnetization effects at $1.7 \mathrm{~A}$ in the quadrupole, and dipole coils.

It is also expected that there will be residual iron yoke magnetization effects. The superconductor persistent currents could be cleared by coil heating above the NbTi $9 \mathrm{~K}$ critical superconductor temperature. For that reason each coil has a heater. The iron residual magnetization could be suppressed by proper degaussing. The iron yoke will be assembled from properly annealed low carbon steel, AISI 1006 or AISI 1008, having a low coercive force.

\section{CONCLUSION}

The proposed variants of compact magnet package designs are needed for medium energy range Linear Accelerators which are under the design now. The magnet package combines the quadrupole and dipole correctors. The main advantages of the proposed design are:

- Splittable configuration allows to install the magnet out of a very clean room;

- Conduction cooling, and cryogen-free magnet design (no LHe vessels for the magnet and current leads);

- Iron dominated magnetic design is less sensitive to the coil geometry and position.

- Coils far away from the magnet aperture reduce superconductor magnetization effects.

- The simple magnet configuration is very efficient for serial magnet fabrication.

A $3 \mathrm{~T}$ integrated gradient quadrupole magnet prototype with combined quadrupole and dipole coils will be tested soon with the main goal to investigate the influence of superconductor magnetization effects on the field quality at low currents.

\section{REFERENCES}

[1] "International Linear Collider Technical Design Report," June 2013, http://www.linearcollider.org/ILC/Publications/Technical-DesignReport

[2] “TESLA Design Report,” Hamburg, DESY 2001-11, 2001.

[3] "Fermilab Proton Improvement Plan," PIP-II, 2013: http://www-ad.fnal.gov/proton/PIP/PIP_index.html

[4] V.S. Kashikhin, N. Andreev, Y. Orlov, D. F. Orris, and M. A. Tartaglia, "Superconducting Magnets for SCRF Cryomodules at Front End of Linear Accelerators," Proceedings of IPAC'10, Kyoto, Japan, 2010, pp. 379-381. 
[5] V. S. Kashikhin, N. Andreev, J. Kerby, Y. Orlov, N. Solyak, M. Tartaglia, and G. Velev, "Superconducting splittable quadrupole magnet for linear accelerators," IEEE Trans. Appl. Supercond., vol. 22, no. 3, p. 4002904, June 2012.

[6] N. Kimura, N. Andreev, V. S. Kashikhin, J. Kerby, M. A. Tartaglia, and A. Yamamoto, "Cryogenic performance of a conduction cooling splittable quadrupole magnet for ILC cryomodules," Adv. Cryogenics Engineering, 59A, 2014, pp.407-415.

[7] R. Carcagno, et al, "Magnetic and Thermal Performance od a Conduction-Cooled Splittable Quadrupole," IEEE Trans. Appl. Supercond., vol. 24, no. 3, p. 4001604, June, 2014.

[8] N. Andreev, V. S. Kashikhin, J. Kerby, N. Kimura, M. Takahashi, M. A. Tartaglia, T. Tosaka, and A. Yamamoto, "Conduction cooling test of a splittable quadrupole for ILC cryomodules," IEEE Trans. Appl. Supercond., vol. 23, no. 3, p. 3500305, June, 2013.

[9] V.S. Kashikhin, et al., "Test results of a superconducting quadrupole model designed for linear accelerator applications," IEEE Transactions on Applied Superconductivity, vol. 19, Issue 3, Part 2, June 2009, pp. 1176-1182.

[10] F. Toral,_et al., "Magnetization Effects on the Superconducting Combined Magnet Prototype for XFEL"", IEEE Transactions on Applied Superconductivity, vol. 19. No. 3, June 2009, pp. 1136-1140.

[11] A. Koski, R. Bandelmann, S. Wolff, "Superconducting magnet package for the TESLA test facility," IEEE Transactions on Magnetics, vol. 32, No. 4, July 1996, pp. 2155-2158.

[12] V. V. Kashikhin, A. V. Zlobin, "Correction of the Persistent Current Effect in $\mathrm{Nb}_{3} \mathrm{Sn}$ Dipole Magnets," ASC2000, IEEE Transactions on Applied Superconductivity, Vol. 11, No. 1, March 2001, pp.2058-2061. 